\title{
LA TRADUCCIÓN DEL INFERNO DE PEDRO FERNÁNDEZ DE VILLEGAS: LA HUELLA DE LA TRADICIÓN POÉTICA CASTELLANA Y DE LOS COMENTARIOS A LA COMMEDIA DE DANTE ${ }^{1}$
}

\author{
PEDRO FERNÁNDEZ DE VILLEGAS' TRANSLATION OF \\ THE INFERNO: THE INFLUENCE OF THE CASTILIAN POETIC \\ TRADITION AND MEDIEVAL COMMENTARIES ON \\ DANTE'S COMMEDIA
}

\author{
MARTA MARFANY \\ Universitat Pompeu Fabra
}

\begin{abstract}
Resumen: A comienzos del siglo XVI, el arcediano de la catedral de Burgos Pedro Fernández de Villegas llevó a cabo, por encargo de Juana de Aragón -hija natural de Fernando el Católico-, una traducción en coplas de arte mayor del Inferno de Dante, que fue impresa en 1515 acompañada de un comentario. El artículo presenta las principales características de la traducción, que se explican a la luz de la tradición poética castellana (especialmente Juan de Mena) y de la tradición exegética medieval de la obra de Dante (Cristoforo Landino, L'Ottimo commento o Benvenuto da Imola). Secundariamente, también se ofrece una visión general sobre la figura de Juana de Aragón, comandataria y destinataria de la traducción.
\end{abstract}

Palabras clave: traducciones medievales; traducción de poesía; Fernández de Villegas; Cristoforo Landino; comentarios a la Commedia de Dante; Juan de Mena; Juana de Aragón.

\begin{abstract}
At the turn of the sixteenth century, the archdeacon of Burgos cathedral Pedro Fernández de Villegas undertook a translation of Dante's Inferno in coplas de arte mayor. Commissioned by Juana de Aragon -Fernando el Católico's natural daughter-, this translation was printed in 1515 and was accompanied with a commentary. The article discusses the main features of the translation, which are analysed in the light of the Spanish poetic tradition (especially Juan de Mena) and of the medieval exegetical tradition of Dante's work (Cristoforo Landino, L'Ottimo commento or Benvenuto da Imola). A portray of Juana de Aragon, mandator and recipient of the translation, is also provided.
\end{abstract}

Keywords: medieval translations; poetry translation; Fernández de Villegas; Cristoforo Landino; commentaries on Dante's Commedia; Juan de Mena; Juana de Aragón.

${ }^{1}$ Este trabajo se ha elaborado en el marco del proyecto FFI2014-53050-C5-4-P (Universitat Autònoma de Barcelona) del Ministerio de Economía y Competitividad. 


\section{SUMARIO}

1. Introducción.- 2. Juana de Aragón, comandataria y destinataria de la traducción.3. El comentario.- 4. La traducción: Dante en coplas de arte mayor.- 5. Los comentarios a la Commedia y la tradición poética castellana: Dido, Aquiles y el episodio de Paolo y Francesca como ejemplos.- 6. Conclusiones.- 7. Bibliografía citada.

\section{INTRODUCCIÓN}

Se conservan en lengua castellana cuatro traducciones antiguas de la Commedia de Dante, todas ellas parciales: la traducción en prosa del Inferno atribuida a Enrique de Villena, de 1428; una traducción anónima en verso conservada en un manuscrito de la segunda mitad del siglo XV, que se limita al primer canto del Inferno y a un terceto del canto XXVII (vv. 22-24); la traducción de Hernando Díaz, impresa en Sevilla en 1516, que traduce en coplas de arte mayor el inicio del primer canto del Infierno, del Purgatorio y del Paraíso (los doce versos iniciales de cada primer canto) ${ }^{2}$; y, finalmente, la traducción del Inferno de Pedro Fernández de Villegas, sobre la cual versará este artículo, escrita también en coplas de arte mayor, conservada en un manuscrito de principios del siglo XVI e impresa en Burgos en 1515 acompañada de un extenso comentario en prosa del propio Villegas.

Hasta hace muy poco, la traducción y el comentario de Villegas no habían recibido suficiente atención por parte de la crítica. No obstante, en estos últimos años, afortunadamente el panorama ha cambiado y hoy ya contamos con varios estudios importantes ${ }^{3}$. Cabe destacar los trabajos de Cintia Maria Hamlin, que han permitido esclarecer, entre otros muchos aspectos, algunas de las cuestiones cruciales sobre la fecha de la traducción y del comentario, por un lado, y sobre la relación entre el manuscrito y la edición de Burgos, por el otro ${ }^{4}$. Ante la visión amplia y detallada que nos ofrecen estos trabajos, el presente artículo se limitará, pues, a puntualizar e ilustrar algunas cuestiones de detalle sobre la traducción relativas a la adaptación métrica que conciernen, por una parte, a la tradición poética del traductor y, por otra, a su conocimiento de la tradición exegética dantesca. Asimismo, se insistirá en

\footnotetext{
2 Para la traducción de Enrique de Villena, véase Pascual 1974; para la traducción anónima, Penna 1965; y, para la traducción de Hernando Díaz, véase Selig 1960. Para una descripción general de los manuscritos o impresos que contienen dichas traducciones, con bibliografía específica, véase, respectivamente Proyecto Boscán, números de registro 653, 652 y 2259.

${ }^{3}$ A los primeros estudios destacados, llevados a cabo por M. Andreu (Andreu 1995, 1998), se han sumado ahora el libro de R. Mondola (Mondola 2011), y varios estudios de C.M. Hamlin (Hamlin 2012a, 2012b, 2012c, 2012d, 2013a, 2013b), entre otros.

${ }^{4}$ Hamlin 2013a.
} 
la figura de Juana de Aragón y su papel de estímulo cultural, especialmente en la Corona de Aragón durante su etapa barcelonesa.

Pedro Fernández de Villegas (1453-1536), eclesiástico burgalés, doctor en Teología, vivió en Roma entre los años 1485 y 1490, y ocupó, a partir de 1496, el cargo de arcediano de la catedral de Burgos. Llevó a cabo su traducción entre 1502 y 1510, mientras que el comentario lo elaboró posteriormente, entre 1510 y $1515^{5}$. El manuscrito de su versión de la Commedia (Hispanic Society of America, B2183) contiene la traducción completa en verso del Infierno, casi idéntica a la versión que se imprimió en Burgos en 1515 acompañada de un extenso comentario. Tradicionalmente se había considerado que el manuscrito había sido la base para la edición de Burgos, pero los trabajos recientes de Hamlin, que ha cotejado ambos testimonios, demuestran lo contrario: el manuscrito es posterior a la edición impresa, y debe fecharse entre 1515 y $1524^{6}$.

Cabe también destacar que, según parece, Villegas estuvo a punto de no publicar la obra, tal como explica Hernando Díaz en el prólogo a su traducción: hacia 1512 Hernando Díaz y Fernández de Villegas coincidieron en Burgos y, al saber Villegas que Díaz también estaba trabajando en una traducción de la Commedia, decidió no publicar la suya; no obstante -siempre según Hernando Díaz-, Villegas cambió de opinión al ver que la versión de Díaz se demoraba en salir a la luz? .

\section{JUANA DE ARAGÓN, COMANDATARIA Y DESTINATARIA DE LA TRADUCCIÓN}

En el proemio a su traducción, Villegas explica que la obra fue un encargo de la muy excellente señora doña Juana de Aragón, duquesa de Frías y condessa de Haro, fija del muy poderoso rey don Fernando de Castilla y Aragón, llamado el Cathólico ${ }^{8}$. Juana había nacido a finales de los años sesenta, fruto de una relación de Fernando de Aragón con la hija de un oficial, Joana Nicolau. Fernando reconoció a la niña como hija suya, la incluyó en el testamento y asumió los gastos de su educación, de la cual parece que se

\footnotetext{
${ }^{5}$ Ibidem.

${ }^{6}$ Ibidem.

${ }^{7}$ Hernando Díaz lo explica en el prólogo a su traducción: "una vez nos comunicamos estando la corte en burgos lo qual avra quasi quatro años: tuuo intencion [Villegas] de no publicarla [su traducción]: mi luenga dilacion mudo su proposito" (apud Proyecto Boscán, número de registro 2259).

${ }^{8}$ Fernández de Villegas 1515 , p. 16. En las citas subsiguientes de la traducción y el comentario de Villegas, indico el número de página entre paréntesis inmediatamente después de la cita. También puntúo y acentúo el texto.
} 
encargó Estefania Carrós y de Mur9 . Fue criada, pues, en Barcelona, donde recibió las atenciones que merecía la hija de un rey, aunque no fuera legítima. Con el paso del tiempo, Juana debió de relacionarse con el ambiente cultural de la ciudad de Barcelona, pues el humanista italiano Antonio Geraldini, al servicio de la Corona de Aragón desde 1469, le dedicó una recopilación de odas en latín, publicadas en 1486 en Roma con el título de Carmina ad Iohannam Aragonum $^{10}$. Por otro lado, Juana de Aragón también podría encajar - es solo una hipótesis- con la Joana d'Aragó a quien Francesc Alegre dedica sus Transformacions, traducción al catalán de las Metamorfosis de Ovidio publicada en Barcelona el 1494 pero elaborada años antes (ya terminada en $1482)^{11}$. La proximidad geográfica y cultural de Juana de Aragón, hija natural del rey Fernando, y su perfil de dama culta con intereses literarios nos inclinan a proponerla como destinataria de la traducción de Francesc Alegre en vez de la identificación tradicional con Juana la Loca (1479-1555), que carece de una justificación histórico-cultural sólida ${ }^{12}$. En 1494, cuando se publicó la traducción de Francesc Alegre, Juana de Aragón aún debía de residir en Barcelona, pues su enlace con Bernardino Fernández de Velasco, duque de Frías, conde de Haro y condestable de Castilla -cuya primera esposa murió en 1499-, que la llevará a vivir en Burgos, no se produjo hasta $1502^{13}$.

Sea como fuere, la relación de Fernández de Villegas con Juana de Aragón se inició en Burgos a raíz de su matrimonio con Bernardino Fernández de Velasco y, en efecto, en el prólogo a su traducción de Dante, el arcediano la describe como una dama culta: consiguió no mediocremente las letras latinas y asaz competente introduçión en la lógica y filosofía moral y mucho de los poetas y oradores (p. 16). Villegas explica también que Juana era doctíssima en muchos auctores (p. 16) y que se sabía de memoria muchos fragmentos de la Commedia: paresçe retener a mente la mayor parte del Dante (p. 16). Según se desprende del prólogo, Juana de Aragón quizás había leído algunos de los comentarios de la obra de Dante, porque, además de conocer la Com-

\footnotetext{
${ }^{9}$ Vinyoles, Comes 2004, pp. 42-46.

${ }^{10}$ Véase Früh 2005, pp. 78-81 y 216-287; 2000.

${ }^{11}$ Véase Torró 1994, p. 224.

${ }^{12}$ Un argumento, aunque no probatorio, a favor de la identificación con la hija ilegítima del rey Fernando el Católico es que Francesc Alegre, en la dedicatoria a su traducción, no se refiere a "Joana d'Aragó" como infanta ni tampoco menciona a la reina Isabel: "A la il·lustríssima senyora, la senyora dona Joana de Aragó, filla del molt alt e potentíssim senyor don Ferrando Segon, nostre rey e senyor, ab humil affecció, Ffrancesch Alegre besa les dignes mans"; Duran, Solervicens 1996, p. 34.

${ }^{13}$ Una vez más, debemos la fijación de la fecha de la boda, entre otras cuestiones de datación, a las investigaciones de C. M. Hamlin, que ha corregido el error transmitido por la crítica de considerar el año 1494 la fecha de la boda entre Juana de Aragón y Bernardino Fernández de Velasco. Véase Hamlin 2013a, p. 110.
} 
media, era capaz de citar lo que los grandes autores habían dicho sobre esta obra: del qual [de Dante] muchas autoridades refería (p. 16). Es muy posible que Juana adquiriera gran parte de estos conocimientos durante su formación en Barcelona ${ }^{14}$.

\section{EL COMENTARIO}

Sabiendo, pues, el interés de Juana en la "doctrina" que los comentaristas extraían de los versos de Dante, Villegas confecciona su comentario o glosa incorporando las novedades que había conocido durante su estancia en Roma (de la doctrina suya [de Dante] yo robé alguna pequeña noticia mientras residi en la corte romana, p. 16), principalmente el comentario de la Commedia en italiano de Cristoforo Landino, de 1481, en el cual se basa su texto: el docto y muy elegante Cristóforo Landino (...) al qual yo en esta glosa e interpretación más que a ninguno otro entiendo seguir (p. 16). Pero en realidad el comentario de Villegas no es estrictamente ni una traducción del comentario de Landino ni tampoco una interpretación del Infierno de Dante, sino que su comentario está basado en la traducción, es decir, Villegas comenta el texto castellano, su traducción; por eso, a pesar de que sigue muy de cerca a Landino y a otros comentaristas, su discurso incluye referencias a personajes castellanos y acontecimientos de la época, vivencias personales de su estancia en Roma y, el aspecto más importante, comentarios lingüísticos o estilísticos sobre la traducción, entre muchas informaciones que no aparecen en ningún otro comentario de la Commedia. No me extenderé en ejemplos sobre el comentario de Villegas, pues ya ha sido muy bien estudiado, pero ofreceré algunos ejemplos ilustrativos para situar al lector.

En el primero de ellos, Villegas cita como autoridad Alonso de Madrigal, el Tostado (1400-1455), obispo de Ávila, y su comentario a los libros de la Biblia:

Queste parole di colore oscuro vid'ïo scritte al sommo d'una porta Inf. III, 10-11
Aquestas palabras de un color obscuro vi sobre una puerta de orrible senblança Inf. III, copla 2

COMENTARIO: Sobre una puerta. En muchos logares de la Sagrada Escriptura se faze mención de la puerta del infierno, pero sy

\footnotetext{
${ }^{14}$ Es harto conocida la difusión temprana de Dante en la Corona de Aragón: en 1429 Andreu Febrer tradujo al catalán la Commedia de Dante íntegra y en terza rima. A lo largo del siglo $\mathrm{XV}$, los comentarios a la obra de Dante circularon por la Corona de Aragón y se conserva una traducción parcial al catalán del Commento de Cristoforo Landino.
} 
aya puerta o camino para allá trátalo bien el Tostado en el capítulo XIV sobre sant Matheo, donde él concluye no aver para el infierno puerta ni via ni necessidad ninguna d'ello porque las ánimas no se cierran ni se abren con puertas (...) (p. 90 $)^{15}$.

En el segundo ejemplo, que pertenece al comentario de los versos 67-72 del canto IV, hay una larga digresión histórica que llega hasta Fernando el Católico:

y en nuestra España oy abría arianos y muchos géneros de infieles si no fuera la virtud y fuerça de los claros príncipes Recaredo, Sysebuto y otros, y quedara España por siempre opressa de la infidelidad de Mahoma si no lo estorvaran las gloriosas victorias de los claros reyes Fernandos, Alfonsos, Ramiros y otros excelentes príncipes y cavalleros (...) pues para qué fablaremos de los passados, teniendo presente al muy poderoso rey y señor nuestro Don Fernando el Cathólico, vuestro padre, muy excelente señora, de quien por todos los tiempos venideros fasta el fin del mundo no faltarán perpetuos loores: ganó el reino de Granada de los moros con tantas y tan gloriosas victorias y fizo convertir a la fe cathólica toda aquella morisma

(...) limpiada España de la espurcicia mahomética, él y la gloriosa reyna Doña Isabel, de eterna memoria, su muger y señora nuestra, echaron y alcançaron de toda España la pestífera muchedumbre de los judíos (...)

Comentario a Inf. IV, copla 14 (p. 129)

También encontramos referencias a obras propias, como un tratado para clérigos y un resumen sobre la historia del reino de Nápoles:

Una de las fijas de la sobervia, como declaramos en nuestro tratadillo de los clérigos, llamado Flosculus sacramentorum, es la presumción.

Comentario a Inf. II, cobla 5 (p. 68)

segund que yo ove scripto más largamente a la reyna nuestra señora Doña Isabel, de gloriosa recordación, al tiempo que con maravillosas victorias se ganó el reyno de Nápoles, faziendo relación a su real magestad de todos los poseedores de aquel reyno y cosas grandes y estrañas acaescidas en él des del año de mill y dozientos fasta estonces.

Comentario a Inf. X, cobla 19 (p. 301)

${ }^{15}$ Las citas al texto original de Dante provienen de Dante 1994. 
Villegas menciona incluso su fecha de nacimiento y los acontecimientos históricos de la época en relación al inicio del viaje de Dante, que sitúa un 25 de marzo:

Yo, Pero Fernández de Villegas, interpretador muy inorante deste poeta, tengo mayor causa de devoción y de ser en él más devoto regraciador a Dios porque en tal día nascí, a XXV de março, día de la anunciación de Nuestra Señora, año de mill y quatrocientos y cinquenta y tres, que fue tiempo muy señalado de turbaciones en esta cibdad de Burgos. Fue mi padrino (...) Alonso Pérez de Vivero, contador mayor, y luego el día siguiente le fizo matar el maestre de Santiago, Don Álvaro de Luna, por lo qual el rey Don Juan II (...) fizo prender al dicho maestre y (...) por este caso y por otros que hera avido por culpado lo mandó cortar la cabeça en Valladolid.

Comentario a Inf. I, copla 6 (p. 34)

A propósito de Cicerón, Villegas explica que, cuando estaba en Roma, tuvo ocasión de presenciar la apertura de la tumba de Tuliola:

Tuvo este Tulio una fija llamada Tuliola que casó con un noble romano nombrado Dolobela; murió muy moça. Fue fallada y abierta la sepultura desta el año de mill y quatrocientos y ochenta y cinco, que fue el primer año del pontificado de Inocencio octavo. Tenía una cobertura de cierta confación como una costra de que toda ella estaba cubierta con la qual se conservó aquel cuerpo sin ninguna corrupción en la mesma manera que el día que murió. Traxéronla a Roma de donde fue fallada, que era el monasterio de las Tres Fontanas, y yo la vi en el Capitolyo, que residía yo estonces en la corte romana.

Comentario a Inf. IV, copla 27 (p. 146)

Son numerosos los comentarios lingüísticos sobre su traducción. Reproduzco uno como ejemplo:

ma per trattar del ben ch'i' vi trovai, dirò de l'altre cose ch'i'v'ho scorte.

Io non so ben ridir com'i' v'intrai Inf. I, 8-10
Mas porque se trate del bien que he [fallado y de cosas otras que pude saber, diré lo escrutado maguer mi entender no sabe dezir cómo ally fuese entrado

Inf. I, copla 2

COMENTARIO: Maguer mi entender. Vocablo castellano antiguo es maguer. Agora no es en uso de curiales ni de galanes, queda solamente en labradores y en las montañas, pero yo soy mucho aficionado a vocablos antiguos, y por eso pongo este y otros algunos de su suerte, de qué no dudo seré reprehendido, mas todo es de sufrir por honra de la antigüedad (p. 27) 
También comenta los italianismos que utiliza en la traducción, como en este pasaje:

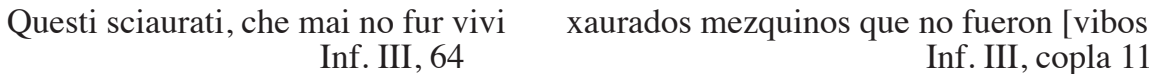

COMENTARIO: que mientra vivieron su vida fue nada ni se entremetieron en fazer mal ni bien ni tovieron motivos que los moviesen a lo uno ni a o otro. Llámalos xaurados, es propio vocablo que pertenesce a estos, por eso se puso asý, italiano como lo es: llaman en Italia a una persona sin consuelo y sin alivio ninguno para fazer nada xaurado, y también las mugeres cuando lloran a sus maridos llámanse xauradas y captiuas en sus lloros que fazen (p.99)

Finalmente, cabe decir también que todas las citas de textos latinos son traducidas directamente al castellano, y la justificación que da Villegas por no citar el original en latín es la siguiente:

Olvidábame de dezir un acuerdo por mi tomado, que creo no será desplazible a los leyentes, y es que en algunas escripturas fechas originalmente por algunos notables auctores o tresladadas de la lengua latina en la nuestra castellana ponen las auctoridades del latín asý en sus palabras latinas, después tórnanlas en castellano, lo qual a mi parescer es muy enojoso al que sabe latín y al que no lo sabe (...) por esto yo, pues esta escritura es en romance castellano, pongo las auctoridades asý en castellano, que a los unos y a los otros puede satisfacer.

Introducción (p. 22)

\section{LA TRADUCCIÓN: DANTE EN COPLAS DE ARTE MAYOR}

En cuanto a la traducción, Juana de Aragón la encargó a Fernández de Villegas con el requisito de que fuera en verso: me mandó vuestra senyoría probase a le [el texto de Dante] trasladar en nuestro vulgar castellano ansí en verso como él estaba (p. 16). Aunque fuera un deseo de Juana, para justificar el uso del verso Villegas cree necesario añadir otros argumentos:

el escrebir versos es obra de entendimiento desocupado, mas como el obedescer a vuestra señoría no me hera arbitrario o voluntario sino subjeto a neçessidad, esforçéme contra mi flaqueza paresciéndome menos malo ser notada de vuestra señoría mi ignorancia que mi desobediençia. (...) quel fazer coblas veo ser tenido en este reyno por acto liviano y de poca autoridad y que por ventura seré notado de no convenir ya tal exerciçio a mi hedad; la cabsa deste herror, que en verdad lo hes, va proçedido de que esta lengua castellana es tan copiosa que todos pueden en ella 
fazer coplas y las fazen buenas o malas, así que la cosa está en manos de quien quiera (...) y lo que peor es que no se fazen coplas sino de vanidades y motes y de amores. De manera que no por culpa del trobar, sino de aquellos que dello mal usan es venido en tan liviana opinión que quien faze coplas es visto fazer cosa de pequeña auctoridad. Mas lo cierto es, muy exçelente señora, que en todas las lenguas la flor dellas (...) es el verso, y ansí en verso fueron escriptos muchos libros de la Sagrada Escriptura (...), y otros muchos, que en la traslación fue trabajoso guardarse la consonançia y no se pudo ansí transferir. (...) la yglesia muchos de los loores de Dios en versos los canta, en hymnos, en prosas consonantes. Desvarío es perder tiempo en confutar tan vana y vulgar opinión, pues coplas castellanas, ¿quántos gravíssimos varones las escrivieron? Don Iñigo López de Mendoza, visaguelo de los señores de vuestra magnífica casa de Velasco; el grave y dotíssimo Juan de Mena; Fernán Pérez de Guzmán, Gómez Manrique, Don Alfonso de Cartajena, obispo de Burgos; y otros gravíssimos auctores. Pues quédese el loable exerçicio del trobar en su debida auctoridad y culpados los que para sus vanidades mal lo emplean.

Proemio (p. 17)

La defensa de la poesía con base bíblica ya la había llevado a cabo Iñigo López de Mendoza, marqués de Santillana, en su Prohemio (procedente de la Genealogia XIV de Boccaccio). Pero además de justificar la elección del verso, en este pasaje Fernández de Villegas se posiciona en una determinada línea de la tradición lírica castellana. Es este uno de los motivos por los cuales no reproduce la métrica del original italiano, la terza rima (tercetos endecasílabos con rima encadenada), sino que su traducción es en coplas de arte mayor, estrofas de ocho versos dodecasílabos que riman ABBA-ACCA, con dos hemistiquios de seis sílabas. El traductor confiesa haber empezado a traducir en terza rima y argumenta por qué se echó atrás: Yo prové a los fazer [los versos] ansí en tercetos, la cual manera no es en nuestro uso y parecíame una cosa tan desdonada que lo dexé (p. 20). La elección métrica de Villegas, que puede parecer al menos curiosa desde una óptica moderna, responde a los procedimientos de traducción de poesía entre lenguas vulgares habituales en el siglo XV: escribir en verso, aunque fuera una traducción, implicaba el uso de unas formas métricas limitadas y concretas que marcaba la tradición poética del traductor, es decir, la métrica del original era adaptada a una estrofa y un verso de uso habitual en la poesía de la lengua meta de la traducción. Y, en la tradición poética castellana, la introducción del verso italiano de Boscán y Garcilaso aún quedaba lejos ${ }^{16}$. Villegas, pues, justifica así su elección:

${ }^{16}$ Véase, para la adaptación de la métrica en otras traducciones de los siglos XV y XVI, al catalán y al castellano, Marfany 2013. 
[La versificación de Dante es] conforme al trobar castellano de arte mayor en que Juan de Mena escrivió el su Laberinto de las trezientas coplas y, porque aquella manera es tan conforme al verso suyo y tanbién porque es mas grave y de mayor resonancia como convenía a tan grave auctor, yo fize esta traslación en aquella forma de trobar que propiamente es verso heroico.

Introducción (p. 20)

En efecto, las coplas de arte mayor eran la forma métrica que los poetas castellanos habían utilizado para tratar cuestiones profundas o, para decirlo con las palabras de Villegas, temas graves y heroicos. Además, habían escrito en coplas de arte mayor, por ejemplo, el marqués de Santillana (13981458) y Juan de Mena (1411-1456), máximos representantes de la llamada escuela alegórico-dantesca del siglo XV castellano ${ }^{17}$. En la elección métrica del traductor, como él mismo indica, fue determinante el Laberinto de Fortuna o Las trescientas de Juan de Mena: escrita en los años cuarenta del siglo $\mathrm{XV}$, esta obra tenía plena vigencia en tiempos de Villegas y a lo largo del siglo XVI, hasta el punto de que fue a su vez objeto de glosas y comentarios como los que se hacían sobre la Commedia de Dante -Villegas podría haber conocido el comentario al Laberinto de Fortuna de Hernán Núñez ${ }^{18}$-. Como en las otras traducciones en verso del siglo XV y principios del XVI, uno de los objetivos que guía la traducción de Villegas es la elaboración de un poema bien hecho en la lengua meta, que siga las normas métricas y estilísticas de su tradición. Las coplas de arte mayor, asociadas a cuestiones elevadas, fijadas y consolidadas por autores que habían recibido la influencia de Dante, eran el medio perfecto para una traducción de la Commedia. No es extraño, pues, que la traducción de Hernando Díaz también sea en coplas de arte mayor. Por otro lado, para Villegas, Juan de Mena era, además de un modelo de versificación, un modelo de lengua y estilo, como insiste muchas veces en el comentario, per ejemplo en un verso del canto octavo, ca dentro no le oyen por bien que razona:

COMENTARIO: ca allá no le oyen por bien que razona. (...) Vocablo castellano antiguo es aquel $c a$; quiere decir "porque", y así se falla en los libros y escripturas castellanas antiguas. Mucho le usa

\footnotetext{
${ }^{17}$ También se incluye en este grupo Diego de Burgos, que estuvo al servicio del marqués de Santillana y que, al morir este en 1458, escribió en coblas de arte mayor el Triumfo del Marqués, donde Dante hace de guía al poeta por un infierno de sabios. Fernández de Villegas era pariente de Diego de Burgos (Arce 1984, p. 193).

${ }^{18}$ El texto de Juan de Mena con glosas de Hernán Núñez fue impreso por primera vez en 1499 y se publicó una segunda edición corregida en 1505 -de la cual derivan todas las ediciones posteriores-; en 1582 aparecía la versión del Laberinto de Fortuna anotada por el Brocense. Para estas cuestiones, véase Mena 1994, pp. LXXXV-LXXXVI.
} 
Juan de Mena y otros elegantes auctores, y es muy bueno y breve, especialmente para en verso.

Inf. VIII, cobla 20 (p. 253)

A pesar de la familiaridad del traductor con las coplas de arte mayor y con la obra de Juan de Mena, traducir Dante a un esquema métrico tan diferente no era un trabajo fácil. Adaptar la terza rima a coplas de arte mayor implicaba un grado elevado de intervención del traductor en el texto, pues el traslado más o menos literal verso a verso era imposible porque los tercetos de Dante no podían cuadrar en una estrofa de ocho versos. De este problema era plenamente consciente el traductor, tal como expone en la introducción: como [Dante] escrive de tres en tres, en dos tercetos faltan dos pies [versos] para una copla del arte mayor (p. 20); y la solución para los dos versos que le faltan es la siguiente:

yo acordé de los suplir desta manera: que algunas vezes, ocurriendo de mio algund buen pie que más aclare su testo o confirme su sentencia, póngole -y aya paciencia el Dante que en su brocado se ponga algund remiendo de sayal que más le faga luzir (...)-; otras vezes suplo aquellos pies de lo que alguno de sus glosadores dize.

Introducción (p. 20)

Por lo tanto, el traductor añade versos de su propia cosecha, ya sea de creación personal, ya sea inspirados por la lectura de los comentaristas de Dante. Al final de la introducción, Villegas declara que, a pesar de estos condicionantes, ha intentado traducir literalmente les palabras de Dante siempre que le ha sido posible: en todo quanto de las mesmas palabras se puede usar, se faze (p. 21), pero puntualizando de nuevo que a veces ha tenido que reproducir el sentido y intención más que no las palabras mesmas (p. 21).

Así pues, la estructura métrica y estrófica de las coplas de arte mayor determina dos tipos de ampliaciones: por un lado, los versos invención "pura" del traductor y, por el otro, los versos sugeridos por la tradición exegética dantesca que Villegas tan bien conoce. En cuanto a la métrica, de manera general, podemos observar el gran influjo que ejerce en la manera de traducir de Villegas ya en el primer verso del poema de Dante: así, In mezzo del cammin di nostra vita es traducido por En medio el camino que va nuestra vida; este añadido que va sirve para sumar dos sílabas, tal como explica el propio Villegas en su comentario a estos versos: Esta palabra "que va" no es del testo del Dante, mas púsose por fazer el verso (p. 24). Similar es lo que sucede en la traducción del verso 33 del primer canto del Infierno, donde se describe la pantera que simboliza la lujuria, che di pel macolato era coverta ("cubierta por una piel moteada"); Villegas traduce su piel hera varia pintada de obscuro, y en el comentario 
explica por qué ha introducido la palabra obscuro: No pone el testo del Dante esta palabra "obscuro": púsose por fazer consonante y por ser propio al caso (p. 33); es decir, usa la palabra obscuro, que no aparece en el texto de Dante, porque necesita una palabra que rime (que haga rima consonante) con duro, que es la palabra rima castellana anterior. También en el comentario de un verso del segundo canto, aunque es al deseo mi fuerza no ygual, aclara: No es del texto del Dante este pie, púsose por cumplir la copla. La traducción de Villegas rebosa de vocabulario, expresiones o versos enteros que no remiten a ninguno del original dantesco y que el traductor se ve forzado a introducir por imperativos métricos. Es en la elaboración de los versos "inventados" para rellenar la copla cuando, en la memoria de Villegas, entran en juego los comentarios leídos de la Commedia y, por el estímulo de la métrica, la tradición lírica castellana.

\section{LOS COMENTARIOS A LA COMMEDIA Y LA TRADICIÓN POÉTICA CASTELLANA: DiDO, AQUILES Y EL EPISODIO DE PAOLO Y FRANCESCA COMO EJEMPLOS}

Para ejemplificar el peso de los comentarios a Dante y de la lírica castellana en la traducción de Villegas, analizaremos dos pasajes del canto V del Infierno, quizás el más apreciado de la Commedia a lo largo de la historia. En el círculo de los lujuriosos, Dante encuentra personajes literarios o históricos que pecaron per exceso de pasión: Semíramis, Dido, Cleopatra, Paris y Helena, Aquiles y Tristán. Los versos que nos interesan en primer lugar son los que conciernen a Dido y a Aquiles:

L'altra è colei che s'ancise amorosa, e ruppe fede al cener di Sicheo; poi è Cleopatràs lussurïosa. Elena vedi, per cui tanto reo tempo si volse, e vedi 'l grande Achille, che con amore al fine combatteo. Inf. V, 61-66
Aquella que veys que se mata amorosa, y rompe la fe al amado Sycheo de su muerte a Eneas yo fágole reo, aunque otros auctores ser niegan tal cosa. Después Cleopatra la tan luxuriosa, Helena sangrienta en millares de miles. Y vimos después a aquel grande Archiles a quien Polícena fue tanto dañosa.

Inf. V, copla 11

Los cuatro primeros versos castellanos, que se corresponden a la primera terzine del ejemplo, hacen referencia a Dido y reflejan una de las polémicas estrella del siglo XV en torno a la Guerra de Troya: en efecto, el episodio del libro IV de la Eneida de Virgilio, en que se relata la trágica pasión de Dido y Eneas, suscitaba partidarios de la "verdad histórica" -no era verosímil que Dido y Eneas coincidieran en el tiempo- y defensores de la "ficción poética" de Virgilio, que entrañaba enseñanzas psicológicas y mora- 
les ${ }^{19}$. Landino, en su comentario, se limita a presentar la versión "histórica" sobre Dido y la "ficción poética" de Virgilio, sin tomar partido por una u otra, introduciendo así la versión virgiliana: Ma Virgilio per ornare el suo poema finge che (...) (Inf. V, 61-63). Pero la frase de Landino se convierte, en el texto de Villegas, en una defensa de la Dido "histórica": El Virgilio por ornar su obra se acordó de infamar a esta tan casta muger diziendo que (...) (Inf. $\mathrm{V}$, copla 11, p. 164). Además, aunque Villegas resume la historia virgiliana de Dido siguiendo a Landino, añade un extenso alegato en contra de la ficción de la Eneida:

El Eusebio dize que no fueron en un tiempo [Dido y Eneas], porque esta Dido fundó aquella cibdad de Cartago CXLIII después de la destrución de Troya. San Hieronymo en una epístola contra Jouiniano dize: Dido, castísima muger, fundó a Cartago y temiendo ser forçada por el rey Hiarba se mató por guardar su castidad. San Agostín, en los libros de la Cibdad de Dios, reprende mucho al Virgilio de aver infamado a tan casta y limpia dueña contra toda verdad, y dize que pecó en ello mucho. Así que bien dize el testo [su propia traducción, copla 11, 4], fablando en nombre de Virgilio ["refiriéndose a Virgilio"] que aunque él faze reo y culpado de su muerte a Eneas, mas que otros auctores dizen lo contrario. El Dante en su testo aquí sigue al Virgilio diziendo lo mesmo de Dido, y aviendo yo visto la auctoridad en contrario destos sanctos doctores Hieronimo y Agostino y de Eusebio, puse en estos dos versos la verdad, y cómo otros doctores de más auctoridad [que yo] dizen el contrario desta culpa levantada y inventada a la casta y limpia Dido.

Comentario a Inf. V, cobla 11 (pp. 164-65)

El propio Villegas explica, pues, la invención del verso aunque otros auctores ser niegan tal cosa, que pone en duda la terzine de Dante con la versión de Virgilio. Las remisiones a Eusebio de Cesarea, san Jerónimo y san Agustín como autoridades no se encuentran en Landino, pero tampoco son originales de Villegas. El cotejo de los principales comentarios a Dante demuestra que el arcediano en este pasaje consultó o tenía en mente la exposición sobre Dido de otros comentaristas de Dante: son los doctores de más auctoridad a los que se refiere en el texto. Eusebio de Cesarea es citado por Boccaccio, y a san Jerónimo y san Agustín remiten Benvenuto da Imola y Francesco da Buti, los tres comentaristas en sus respectivas exposiciones sobre estos mismos versos (Inf. V, 61-63) (el destacado es mío):

\footnotetext{
${ }^{19}$ Fue una de las grandes cuestiones tratadas por distintos autores de la baja edad media, como por ejemplo el marqués de Santillana, quien, en la glosa a Dido de sus Proverbios (v. 428), después de resumir la "verdadera" historia, puntualiza que la versión de Virgilio no debe ser reprobada puesto que se trata de una licencia poética.
} 
La quale oppinione per reverenza di Virgilio io aproverei, se il tempo nol contrariasse. Assai manifesta cosa è Enea il settimo anno dopo il disfacimento di Troia esser venuto, secondo Virgilio, a Didone: e Troia fu distrutta l'anno del mondo, secondo Eusebio, MMMMXX. E il detto Eusebio scrive essere oppinione d'alcuni Cartagine essere stata fatta da Carcedone tirio (...). De' quali tempi alcuno non è conveniente co' tempi d'Enea: e perciò non credo che mai Enea la vedesse.

Boccaccio, Esposizioni sopra la Comedia di Dante, Inf. V, 60-62

Sed hic est attente notandum quod istud, quod fingit Virgilius, nunquam fuit factum, neque possibile fieri, quin Eneas, teste $\mathrm{Au}$ gustino in lib. de Civitate Dei, venit in Italiam per trecentos annos ante Didonem. Ipsa etiam Dido non se interfecit ob amorem laxivum, imo propter amorem honestum (...); fuit enim pudicissima femina, sicut scribit Jeronimus contra Jovinianum hereticum.

Benvenuto da Imola, Comentum super Dantis Aldigherii Comoediam,

Inf. V, 60-62

ma secondo la verità non fu così: imperò che dice santo Girolamo nel primo libro che fece contra Gioviniano, che Dido fu castissima donna, et innanzi volle ardere sè medesima nella pira, et uccidersi, che maritarsi al re Giarba che la voleva per moglie, assegurando che Cartagine fu edificata da castissima donna, et in segno di ciò finie in grandissima castità: (...) e non fu mai vero che Enea capitasse a Cartagine: però che Livio n'avrebbe fatto menzione. Ancora santo Agostino nel primo libro delle Confessioni conferma questo; e però Virgilio fece molto male a dare tale infamia a sì onesta donna, per fare bella la sua poesia; e lo nostro autore Dante fece peggio a seguitarlo in questo, che credo che avesse veduto Geronimo e li altri che di ciò parlano: potrebbesi scusare; ma le scuse non sono sofficienti, però le lascio.

Francesco da Buti, Commento sopra la Commedia, Inf. V, 60-62

Estas coincidencias (referencias a los mismos autores, frases muy parecidas, misma interpretación) demuestran que el arcediano había leído distintos comentarios a Dante y que conocía perfectamente la polémica concerniente a Dido que estos versos suscitaban, así como los distintos posicionamientos de los comentaristas al respecto. Tanto es así que su defensa de la Dido "histórica" le lleva a enmendar a Dante añadiendo en su traducción un verso para desautorizar la historia virgiliana de Dido y Eneas.

La explicación es muy similar para los versos concernientes a Aquiles. Los personajes de Héctor y Aquiles también fueron objeto de numerosos debates literarios, siempre con el trasfondo de la confrontación entre la "ver- 
dad histórica" y la "ficción" que relata Homero ${ }^{20}$, a quien los detractores de Aquiles acusaban de haberlo engrandecido y ensalzado. Villegas, alejándose una vez más de Landino, se muestra en su glosa como ferviente opositor a la figura de Aquiles. Si bien en esta ocasión no se atreve a enmendar el verso de Dante, y el grande Achille es traducido en la copla por el grande Archiles, no obstante, al remitir a este verso en el comentario, lo corrige así: $Y$ vimos después al pérfido Archiles. La justificación del arcediano ante el cambio de adjetivo es la siguiente:

y dize "el pérfido": vocablo latino es, quiere dezir "quebrador de fe", como lo son los que matan a trayción, como este lo fazía; corrigióse este verso que primero dezía "pérfido" y después se puso "grande" porque lo dize el toscano.

Comentario a Inf. V, cobla 11 (p. 165)

La exposición sobre Aquiles de Villegas recoge la idea de que Aquiles mató a Héctor a traición: Este Archiles es el tan loado de fortaleza que mató a Hétor el fuerte (...) aunque le mató a trayción, detalle que en general no recogen los comentarios en este pasaje. Se alude a ello, por ejemplo, en el Ottimo commento, pero solo para refutar esa idea: Dicesi che vi usò tradimento [Aquiles al matar a Héctor], se tradimento si può dire quando l'uno nemico combatte a pieno campo con l'altro (Inf. IV, 122). Los episodios que relata el arcediano de la vida de Aquiles son recurrentes en muchos comentarios y por ello es difícil determinar cuáles conocía. No obstante, hay pequeños detalles que revelan coincidencias significativas con algunos comentarios. Veamos un fragmento del texto de Villegas:

dizen que [murió, por la flecha de Paris] ferido por las plantas de los pies, porque no podía morir de ferida en otra parte, que su madre Tethis le avía metido en la mar con ciertos encantamentos y como le tenía por los pies y no se mojaron podía morir por allý. Significa aquello que los que van y andan en tales passos mueren por los pies que los llevaron allá tras sus afectos y vanos deseos: por eso dize "a quien Polícena fue tanto dañosa".

Comentario a Inf. V, cobla 11 (p. 165)

\footnotetext{
${ }^{20}$ La novela catalana anónima Curial e Güelfa alberga en distintos episodios el debate sobre Héctor y Aquiles. En uno de ellos, el protagonista, en el monte Parnaso, tiene una visión en sueños en la cual debe hacer de juez en un debate a propósito de los dos héroes de la Guerra de Troya, Aquiles y Héctor, y decidir cuál de los dos fue mejor. La sentencia de Curial al respecto es la siguiente: "Jo trobe Hèctor ésser lo millor cavaller que fos entre los troians e Aquil-les lo millor que fonc entre los grecs; e que Hèctor féu més, pus solemnes e majors coses, hac més virtuts e fonc menys viciós; Aquil-les ferí bé a Hèctor, car en batalla cascú deu cercar son avantatge. Homero ha escrit llibre que entre los hòmens de ciència man que sia tengut en gran estima; Dites e Dares escriviren la veritat e així ho pronuncie" (Badia, Torró 2011, p. 437).
} 
En primer lugar, el punto débil de Aquiles es, en la mayoría de comentarios, el talón, calcagno en italiano, y no las plantas de los pies. En segundo lugar, Villegas interpreta metafóricamente los pies como la lujuria, de ahí el verso final de la cobla, tan distinto del original. Son dos aspectos que recoge el comentario de Benvenuto da Imola (el destacado es mío):

Ideo bene fingit Homerus, quod Achilles erat totus invulnerabilis praeter plantas pedum, quia scilicet non poterat superari nisi per viam amoris; nam pes hominis est amor eius, et sic cessat objectio quare autor non nominaverit supra Achillem inter viros illustres, quia Achilles viscatus vicio luxuriae mortuus est turpiter; Hector vero pugnans pro patria mortuus est laudabiliter in praelio.

Benvenuto da Imola, Comentum super Dantis Aldigherii Comoediam, Inf. V, 60-62

Estos ejemplos demuestran, más allá de Landino, el peso de los comentarios a Dante en la confección del texto y la traducción poética de Villegas. El segundo pasaje que analizaremos es también significativo en ese sentido, y además indica la resonancia de la tradición lírica castellana en la obra de Villegas.

Se trata del conocidísimo episodio de Paolo y Francesca, al cual se debe en buena parte la celebridad del canto V. En el círculo de los lujuriosos aparecen también Paolo Malatesta y Francesca da Rímini, contemporáneos de Dante. Francesca, hija del señor de Ravenna, se enamoró de Paolo Malatesta, hermano de su marido, el cual los mató a los dos (el crimen se produjo entre 1283 y 1286 y era, por lo tanto, de plena actualidad cuando Dante escribió la Commedia). Dante hace hablar a Francesca, que explica el momento preciso en que ella y Paolo se enamoraron, mientras leían un libro que explicaba la historia de Lanzarote y Ginebra ${ }^{21}$.

"Noi leggiavamo un giorno per diletto di Lancialotto come amor lo strinse; soli eravamo e sanza alcun sospetto.

Per più fiate li occhi ci sospinse quella lettura, e scolorocci il viso; ma solo un punto fu quel che ci vinse.

Quando leggemmo il disïato riso esser basciato da cotanto amante, questi, che mai da me non fia diviso,
"Entrambos estando en logar apartado de aquel Lanzarote leyendo su historia, el fuego de amor aun en nuestra memoria por actos extrínsecos no demostrado, materia nos dio el lascivo tratado.

De aquellos amantes habiendo leído, suspensos los ojos, cegado el sentido, besó la mi boca tremiendo y turbado.

Ansí Galeoto les fue medianero segund que a nosotros el libro tan vano, en cuya lectura es trabajo liviano

\footnotetext{
${ }^{21}$ En Alvar 1999 se analizan cuatro traducciones castellanas modernas a través de los versos 121-142 del canto V del Inferno. Véase también, sobre el episodio de Paolo y Francesca en las traducciones inglesas del Romanticismo, Saglia 2002.
} 
la bocca mi basciò tutto tremante. Galeotto fu 'l libro e chi lo scrisse: quel giorno più non vi leggemmo avante".

Mentre che l'uno spirto questo disse, l'altro piangëa; sì che di pietade io venni men così com' io morisse.

E caddi come corpo morto cade. Inf. V, 127-142 sin buena doctrina al vevir verdadero." Mientra ella decía, el su compañero . contino lloraba con tanto gemido que su compasión amató mi sentido, y a tierra me lanza el dolor lastimero. Inf. V, coplas 21-22

Los versos de creación propia o elaborados a partir de una frase de algún comentario son fáciles de detectar en este fragmento del episodio de Paolo y Francesca, en el cual Villegas se aleja mucho de les palabras originales de Dante y, en cambio, es cercano al discurso de los comentaristas. De hecho, en estos versos, Villegas, más que traducir, interpreta el original dantesco manteniendo muy pocas palabras de los versos italianos. Así, por ejemplo, en la primera copla, que corresponde a las tres primeras terzine y al primer verso de la cuarta, encontramos pocas coincidencias con el italiano: solo li occhi ci sospinse, que es traducido por suspensos los ojos, y el verso la bocca mi basciò tutto tremante, que es traducido por besó la mi boca tremiendo y turbado. El libro de Lanzarote que leen Paolo y Francesca es, en la traducción, un lascivo tratado que provoca pasión (fuego de amor) y que perturba los sentidos (cegado el sentido), y, en la segunda copla, es llamado el libro tan vano. Además, Villegas amplía el verso 137, Galeotto fu 'l libro e chi lo scrisse, especificando su sentido, igual que hacían los comentaristas: Ansí Galeotto les fue medianero ("Galeoto hizo la función de intermediario [entre Lanzarote y Ginebra]"), según que a nosotros el libro tan vano ("igual que este libro tan frívolo hizo con nosotros"), y añade dos versos de su propia cosecha con un claro contenido moral, en cuya lectura es trabajo liviano / sin buena doctrina al vevir verdadero ("leer este libro es una empresa inútil y sin ningún buen enseñamiento para el vivir auténtico"). Es evidente que, en la traducción de estos versos, Villegas tiene más en la mente los comentarios que no el texto de Dante. Así, por ejemplo, en el Ottimo commento, podemos leer (el destacado es mío):

Finalmente stando l'uno con l'altro (...) e leggendo nella camera della donna un libro della Tavola Ritonda, nel quale era scritto come Lancialotto innamorò della reina Ginevra, e come per mezzana persona, cioè Galeotto Lo-Bruno, Signore de l'Isole lonta$n e$, elli sì congiunse insieme a ragionare di loro amore, e come il detto Lancialotto per virtù di quello ragionamento conosciuto l'amoroso fuoco, fue baciato dalla reina; al quale punto pervegnendo la detta Francesca, vinse la forza di questo trattato sì lor 
due, che posto giù il libro vennero a l'atto della lussuria, al quale diede materia il confortamento di questo libro, sì come Galeotto diede materia a Lancialotto e alla reina.

L'Ottimo Commento, Inf. V, 70-71

En efecto, además de coincidir la interpretación de Villegas con la de este comentario, encontramos coincidencias léxicas: el amoroso fuoco tiene equivalencia en el fuego de amor, el libro de Lanzarote es designado por Villegas como un tratado, igual que en el comentario (questo trattato) y el adjetivo lascivo se puede relacionar con el término lussuria; finalmente, materia nos dio coincide con diede materia, que aparece dos veces en este pasaje del Ottimo commento. La fuente de Villegas en este pasaje se confirma también en su comentario, donde Galeoto es llamado señor de las luengas ínsulas, paralelo al signore de l'isole lontane del Ottimo commento, una denominación usual en la literatura artúrica pero rara en los comentarios de este pasaje de la Commedia:

Fue Galeoto, segund aquel libro de las mentiras de Lançarote y de Tristan y de la Tabla Redonda, un cavallero grande que dezían señor de las luengas ínsulas, mucho amigo de Lançarote.

Comentario a Inf. V, cobla 22 (p. 172)

Por otro lado, cabe destacar también, en la traducción, la omisión del verso quel giorno più non vi leggemmo avante, que seguramente Villegas no trasladó por escrúpulos: en efecto, si lo hubiera traducido de manera literal, el resultado habría implicado, igual que en el original, sugerir, sin nombrarlo, el gran pecado de la lujuria; pero tampoco podía dar la interpretación del verso, porque habría significado nombrar explícitamente ese pecado (el atto de la lussuria del comentario citado). Finalmente, los últimos cuatro versos no se alejan completamente del sentido del texto italiano, pero la dicción es muy diferente: el verbo piangëa se amplifica en contino lloraba con tanto gemido; sì che di pietade / io venni men così com' io morisse se convierte en el verso que su compasión ["mi compasión por él"] amató mi sentido; y el verbo final, E caddi como corpo morto cadde, se adapta, sin ninguna coincidencia léxica ni sintáctica, en y a tierra me lanza el dolor lastimero.

Finalmente, cabe destacar que algunas palabras y expresiones que emplea Villegas sin correspondencia en el texto de Dante encuentran su parangón en la poesía castellana. Por ejemplo, en el Laberinto de Fortuna de Juan de Mena, en un pasaje del círculo de Venus, donde se encuentran los caídos en desgracia por el vicio de la lujuria, hay ciertas coincidencias de vocabulario con el texto citado de la traducción de Villegas: 
Tan grant multitud turbada veyendo

por fuego viçioso de ilíçito amor,

fablé: "Providençia, tú dime mejor

aquesta mi dubda que yo non entiendo;

éstos atanto discretos seyendo,

¿por qué se quisieron amar çiegamente?;

bullada devieran tener en la fruente

la pena que andan aquí padesçiendo"

Juan de Mena, Laberinto de Fortuna, vv. 865-872

Si Villegas, como buen lector de Juan de Mena, tenía en mente este pasaje al traducir el episodio de Paolo y Francesca, no sería insensato proponer que el penúltimo verso, bullada devieran tener en la fruente, quizás le sugirió la cita bíblica con la que glosa los versos de su traducción:

En el capítulo XVII del Apocalipsis dize de aquella putana que tenía escriptos en la fruente los nombres de blasfemia y de Babilon madre de las fornicadoras dize tenerlos escriptos en la fruente manifestándolos y enseñándolos a sus amigos.

Comentario a Inf. V, cobla 22 (p. 173)

Sea como fuere, la expresión dolor lastimero que utiliza Villegas en el último verso del pasaje, sin ninguna equivalencia con el original de Dante, es un estilema que recorre toda la poesía castellana, siempre en posición de rima, como, por ejemplo, en una pieza anónima del Cancionero de Palacio y en una consolatoria de Gómez Manrique:

En fuego de amor me quemo, vivo, muero, desespero y no sé lo que me quiero.

No siento de qué me quexe, siento bien que estoy con quexa, no sé qué tome ni dexe, ni quién me tome ni dexa.

Todo plazer se me alexa, tengo un dolor lastimero y no sé lo que me quiero.

Anónimo del Cancionero Musical de Palacio ${ }^{22}$.

A Este me tornaré

que guaresçe los que hiere;

a Este suplicaré

que graçia y saber me de,

\footnotetext{
${ }^{22}$ Se trata del número 141 de Barbieri. El texto transcrito aquí procede de Iriso 2011, p. 44.
} 
pues que puede lo que quiere

para que pueda comigo

templar como cauallero

este dolor lastimero

y en modo no grosero

hablar, señora, contigo.

Gómez Manrique, Consolatoria para doña Juana de Mendoza ${ }^{23}$

\section{CONCLUSIONES}

En definitiva, la traducción se entiende perfectamente a la luz del entorno cultural e intelectual de Villegas y de su tradición poética ${ }^{24}$. En el siglo XV y a principios del XVI, la operación de escribir en verso, aunque se tratase de una traducción, llevaba implícita la adopción de unas formas métricas limitadas e implicaba la necesaria remisión a una lengua poética, unas fórmulas, unos estilemas y un vocabulario que se imponían en el traductor, ya fuese conscientemente o de manera automática. Por consiguiente, significaba inserir la obra en un código poético diferente, en un sistema que no era el del original sino el de la tradición poética del traductor. Asimismo, puesto que la obra de Dante, además de un texto poético, era un clásico, Fernández de Villegas, emulando a los traductores de los grandes autores latinos, incorporó la interpretación y las palabras de los comentaristas en su traducción.

\section{BIBLIOGRAFÍA CITADA}

Alvar, Carlos (1999), El texto y sus traducciones: a propósito de la Divina Comedia, en Paredes, Juan; Muñoz, Eva (eds.), Traducir la Edad Media. La traducción de la literatura románica, Granada, Universidad de Granada, pp. 135-151.

Arce, Joaquín (1984), Dante y el humanismo español, "Cuadernos para la investigación de la Literatura Hispánica" 6, pp. 184-194.

Andreu, Maribel (1995), La amplificación en el Infierno de Dante traducido por Pedro Fernández de Villegas (Burgos, 1515), Barcelona, Universitat de Barcelona (tesis doctoral).

\footnotetext{
${ }^{23}$ Vidal González 2003, p. 281.

${ }^{24}$ Asociar Dante y los comentarios a la Commedia con Juan de Mena no es un procedimiento exclusivo de la obra de Villegas, pues lo encontramos en poetas como Lluís d'Avinyó, autor en castellano y en catalán. Véase: Galí, Ramos, Torró 2009; Ramos, Rodríguez, Torró 2014.
} 
Andreu, Maribel (1998), Traducir el italiano de Dante en la Castilla del siglo XVI: el Infierno según Pedro Fernández de Villegas, en Orero, Pilar (ed.), Actes del III Congrés Internacional sobre traducció, Barcelona, Universitat Autònoma de Barcelona, pp. 293-302.

Badia, Lola, Torró, Jaume (eds.) (2011), Curial e Güelfa, Barcelona, Quaderns Crema.

Benvenuto da Imola, Comentum super Dantis Aldigherii Comoediam, ed. Jacobo Philippo Lacaita (1887), Florencia, G. Barbèra. Darmouth Dante Project

http://dante.dartmouth.edu/search_view.php?query=\&cmd=Search\&comme ntary $\% 5 \mathrm{~B} \% 5 \mathrm{D}=13755 \&$ language $=$ any $\&$ cantica $=0 \&$ canto $=\&$ line $=$ [consulta: $1 / 2 / 2015$ ]

Boccaccio, Giovanni, Esposizioni sopra la Comedia di Dante, ed. Giorgio Padoan (1965), en Branca, Vittore, Tutte le opere di Giovanni Boccaccio, Milán, Mondadori.

Dante, Alighieri, La Commedia secondo l'antica vulgata, ed. Giorgio Petrocchi (1994), Florencia, Le Lettere.

Duran, Eulàlia; Solervicens, Josep (eds.) (1996), Renaixement a la carta, Barcelona, Eumo.

Fernández de Villegas, Pedro (1515), La traducción del Dante de lengua toscana en verso castellano, Burgos, Fadrique Alemán de Basilea. [Ejemplar consultado: Biblioteca Universitària de València, R-1/158. http://trobes.uv.es/tmp/_webpac2_1354712.3121] [consulta: $1 / 2 / 2015]$

Francesco da Buti, Commento sopra la Commedia, ed. Crescentino Giannini (1858-1862), Pisa. Darmouth Dante Project

http://dante.dartmouth.edu/search_view.php?query=\&cmd=Search\&comme ntary $\% 5 \mathrm{~B} \% 5 \mathrm{D}=13855 \&$ language $=$ any $\&$ cantica $=0 \&$ canto $=\&$ line $=$ [consulta: $1 / 2 / 2015$ ]

Früh, Martin (2000), Los Carmina ad Iohannam Aragonum del humanista italiano Antonio Geraldini (m. 1489): reflexiones preliminares a una edición crítica, "Faventia" 22/1, pp. 141-144.

Früh, Martin (2005), Antonio Geraldini (†1488), Münster, Lid.

Galí, Montserrat; Ramos, Rafael; Torró, Jaume (2009), De mossèn Avinyó a Lluís d'Avinyó, uixer del Príncep de Viana, en Alberni, Anna; Badia, Lola; Cabré, Lluís (eds.), Translatar i transferir. La transmissió dels textos i el saber (1200-1500), Santa Coloma de Queralt, Obrador Edèndum, pp. 475-508.

Hamlin, Cinthia María (2012a), Fernández de Villegas y Landino, traducción y reapropiación, el caso de la dicotomía vida activa/vida contemplativa en el comentario de la Comedia, "eHumanista" 20, pp. 430-450. 
Hamlin, Cinthia María (2012b), El comentario de la Divina Comedia de Fernández de Villegas, características generales y actitudes humanísticas, "eHumanista" 21, pp. 437-466.

Hamlin, Cinthia María (2012c), La traducción en la España pre-humanista y sus causas político-ideológicas: el caso de la Divina Comedia y los reyes católicos, "Revista de Literatura Medieval" 24, pp. 88-100.

Hamlin, Cinthia María (2012d), Perspectivas y planteamientos de una poética: reflexiones sobre poesía y ficción en el comentario a la Divina Comedia de Fernández de Villegas, "e-Spania" 14, pp.1-19.

Hamlin, Cinthia María (2013a), La traducción de la Divina Comedia de Villegas: problemas de datación y filiación de testimonios, "Letras" 67-68, pp. 107-116.

Hamlin, Cinthia María (2013b), La transmisión textual de la traducción de la Divina Comedia (1515), ¿del impreso al manuscrito?, "Revista de Filología Española" 93/2, pp. 273-289.

Iriso, Silvia (2011), El gran libro de los villancicos, Barcelona, El Aleph Editores.

L'Ottimo commento della Divina Commedia, ed. Alessandro Torri, Alessandro (1827-1829), Pisa, N. Capurro. Darmouth Dante Project

http://dante.dartmouth.edu/search_view.php?doc $=133351050700 \& \mathrm{cmd}=$ goto result\&arg1=0 [consulta: 04/08/2014]

Marfany, Marta (2013), Traducciones en verso del siglo XV, "Bulletin of Hispanic Studies" 90/3, pp. 261-273.

Mena, Juan de (1994), Laberinto de Fortuna y otros poemas, ed. Carla de Nigris, Barcelona, Crítica.

Mondola, Roberto (2011), Dante nel Rinascimento castigliano: L'Inferno di Pedro Fernández de Villegas, Nápoles, Tullio Pironti.

Pascual, José Antonio (1974), La traducción de la Divina Comedia atribuida a D. Enrique de Aragón. Estudio y edición del Infierno, Salamanca, Universidad de Salamanca.

Penna, Mario (1965), Traducciones castellanas antiguas de la Divina Comedia, "Revista de la Universidad de Madrid" 14, pp. 81-127.

Proyecto Boscán. Catálogo de las traducciones españolas de obras italianas (hasta 1939) http://boscan.ub.es:591/CATALOGO/FMPro [consulta: $12 / 02 / 2015]$.

Ramos, Rafael; Rodríguez Risquete, Francisco J.; Torró, Jaume (2014), Mossèn Avinyó, the Cancionero de Vindel and the Cançoner llemosí del siglo XV, "Digital Philology. A Journal of Medieval Cultures" 3/1, pp. 142-161.

Saglia, Diego (2002), Translation and Cultural Appropriation: Dante, Paolo and Francesca in British Romanticism, "Quaderns. Revista de traducció" 7, pp. 95-119. 
Selig, Karl Ludwig (1960), The Dante and Petrarch Translations of Hernando Díaz, "Italica" 37/3, pp. 185-187.

Torró, Jaume (1994), "Officium poetae est fingere": Francesc Alegre $i$ la Faula de Neptuno i Dyana, en Badia, Lola; Soler, Albert (eds.), Intel-lectuals i escriptors a la baixa Edat Mitjana, Barcelona, Curial - Publicacions de l'Abadia de Montserrat, pp. 221-241.

Vidal González, Francisco (ed.) (2003), Gómez Manrique, Cancionero, Madrid, Cátedra.

Vinyoles, Teresa; Comes, Mireia (2004), Estefania Carrós y de Mur, Madrid, Ediciones del Orto.

Fecha de recepción del artículo: septiembre 2014

Fecha de aceptación y versión final: febrero 2015 